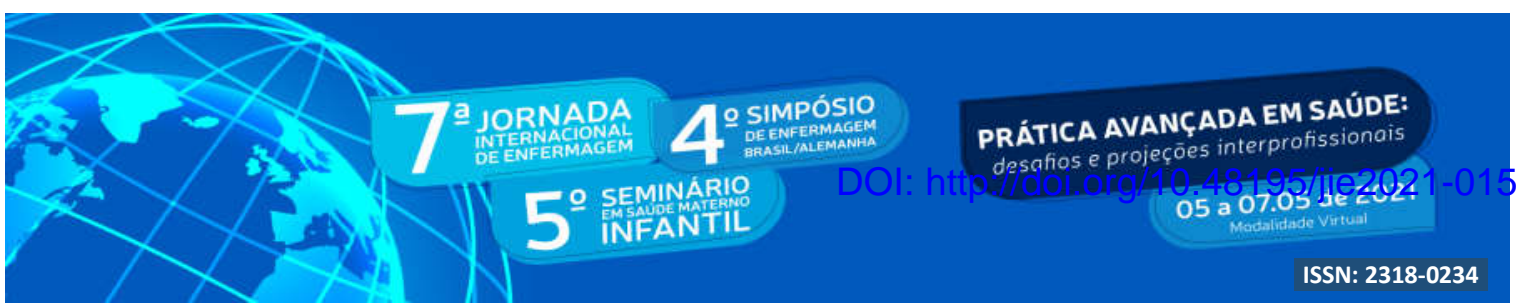

\title{
ASSISTÊNCIA FARMACÊUTICA: PAPEL COLABORATIVO NAS PRÁTICAS DE ATENDIMENTO NOS CENTROS DE ATENÇ̃̃O PSICOSSOCIAL INFANTIL ${ }^{1}$
}

\author{
Michele da Silva ${ }^{2}$; Camila Franco ${ }^{3}$
}

\begin{abstract}
RESUMO
Transtornos mentais na infância e adolescência possuem uma incidência de $12,6 \%$ e configuram elevado número de pacientes e investimentos em saúde na população brasileira, entretanto, o atendimento, diagnóstico e acompanhamento terapêutico é realizado com dificuldades, acarretando em polifarmácia, interações medicamentosas e efeitos adversos. $O$ presente trabalho investigou na literatura os procedimentos e prescrições realizadas nos Centros de Atenção Psicossocial Infantil, de forma a colaborar com a melhoria do serviço e alertar sobre manejos terapêuticos nas situações mais graves de interações medicamentosas. Com esse estudo, notou-se a importância de incluir os aspectos sociodemográficos, histórico familiar e questões religiosas, entre outras, no levantamento de dados do paciente durante acolhimento no serviço. Observou-se também a necessidade de o farmacêutico participar da equipe multiprofissional dos Centros de Atenção Psicossocial, pois cabe a ele o acompanhamento farmacoterapêutico.
\end{abstract}

Palavras-chave: Criança; Classificação Internacional de Doenças; Psicofármacos; Serviços de saúde mental; Transtornos Mentais.

\begin{abstract}
Mental disorders in childhood and adolescence have an incidence around 12,6\% and constitute a high number of patients and investments in health of the Brazilian population, however, the care, diagnosis and therapeutic accompaniment is carried out with difficulties, leading to polypharmacy, drug interactions and adverse effects. The present work investigated in the literature the procedures and prescriptions performed in the Child Psychosocial Care Centers in order to collaborate with the improvement of the service and to alert about the therapeutic management in the most serious situations of drug interactions. With this study, it was noted the importance of including sociodemographic aspects, family history, religious issues, among others, in the collection of data from the patient when he is admitted to the mental health service. It was also observed the need for the pharmacist to be included in the multiprofessional team of the Centers for Psychosocial Care, as it is the responsibility of the pharmaceutical care.
\end{abstract}

Key Words: Child; International Classification of Diseases; Mental Disorders; Mental Health Services, Psychotropic Drugs.

\footnotetext{
${ }^{1}$ Trabalho final de graduação.

${ }^{2}$ Estudante do Curso de Farmácia. Universidade Franciscana. E-mail: micheledasilva804@gmail.com

${ }^{3}$ Orientador. Doutor. Universidade Franciscana. E-mail: cf@ufn.edu.br
} 


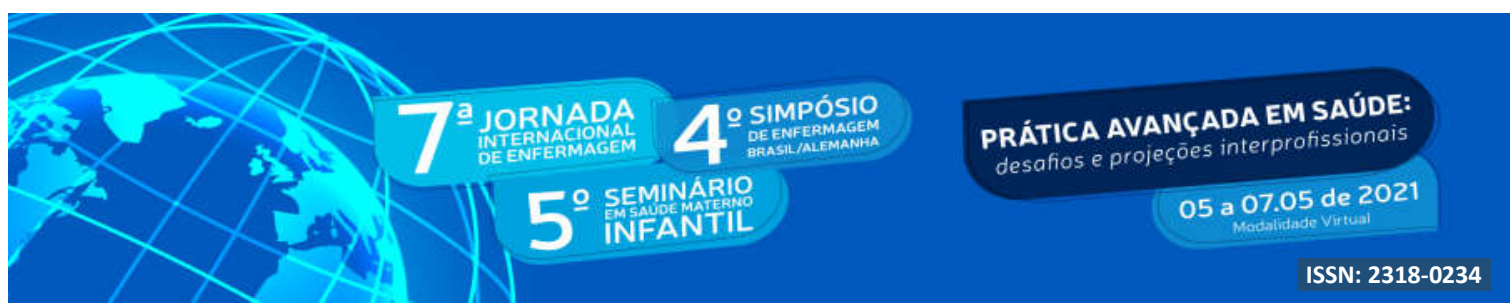

\section{INTRODUÇÃO}

No Brasil 23 milhões de pessoas (12\%) necessitam de algum atendimento em saúde mental, sendo as doenças mais prevalentes a depressão, ansiedade e transtornos de ajustamento (LOURENÇO, 2010). A Lei no 10.216 (06/04/2001) e as Conferências Nacionais de Saúde e Saúde Mental geraram o término do modelo manicomial e a criação de redes de atenção à saúde mental e de leitos psiquiátricos hospitalares. Surge o Centro de Atenção Psicossocial (CAPS) para acolhimento de adultos e, para cuidados de pacientes infantojuvenil, o Centro de Atenção Psicossocial Infantil (CAPSi) (CUNHA, 2017; BRASIL, 2014; HIRDES, 2009).

O CAPSi atende crianças e adolescentes em sofrimento psíquico ou sob uso de substâncias psicoativas. A equipe de saúde é composta por diferentes profissionais, entretanto, a atuação farmacêutica ainda é insuficiente, em horário restrito ou nem mesmo participam da dispensação do medicamento (BRASIL, 2015; TAVARES, 2012). Durante o acolhimento no CAPSi são coletadas informações de sexo, idade, escolaridade, estado civil, trabalho, diagnóstico, queixas e sintomas, entre outros. Nesta curta anamnese, cada profissional prioriza as informações que lhes são mais relevantes, o que dificulta traçar um perfil do usuário em saúde mental (VIEIRA, 2013). O tratamento, na maioria dos transtornos, é baseado na politerapia. Com isso, a prescrição e a interação entre medicamentos devem ser avaliadas de forma criteriosa pelo farmacêutico que poderá intervir mudanças de procedimentos e protocolos referentes ao Uso Racional de Medicamentos (URM) e Problemas Relacionados aos Medicamentos (PRM), colaborando com as práticas de acompanhamento farmacoterapêutico (THIENGO, 2014).

\section{OBJETIVO}

O objetivo deste trabalho é revisar a literatura quanto ao atendimento em saúde mental nos CAPSi para colaborar com os protocolos de atendimento do paciente, diagnóstico e acompanhamento/registro de terapias utilizadas pelo paciente, baseado em adaptações do método Dáder e assim, auxiliando nas práticas do serviço.

\section{METODOLOGIA}




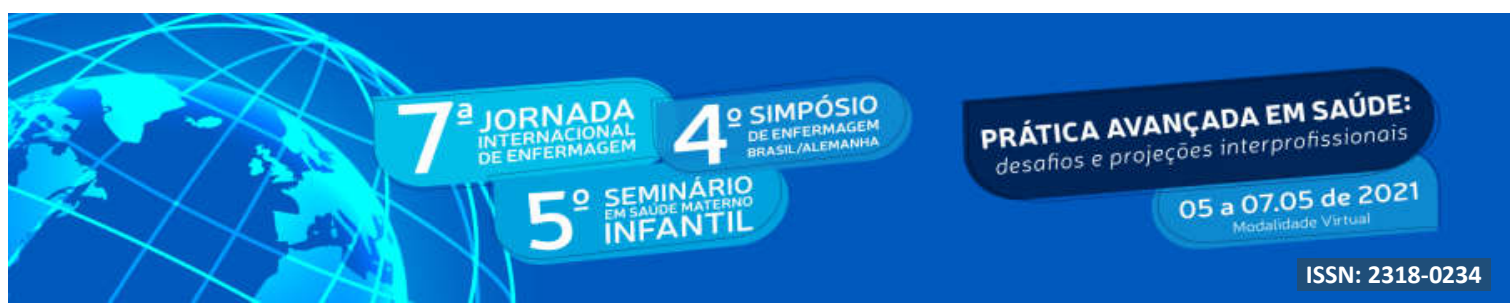

Trata-se de uma revisão de literatura considerando atendimento e prescrições no âmbito dos CAPSi, entre 2008 e 2018, por consulta em Biblioteca Regional de Medicina (BIREME), Literatura Latino-Americana e do Caribe em Ciências da Saúde (LILACS) e Scientific Electronic Library Online (SciELO) utilizando como descritores as palavras-chave. Após a análise dos resultados foram propostas adaptações do protocolo de atendimento do paciente e acompanhamento farmacoterapêutico.

\section{RESULTADOS E DISCUSSÃO}

Das 5 milhões de crianças e adolescentes diagnosticados com doença mental, 8,7\% apresentam hiperatividade ou desatenção, 7,8\% dificuldades com leitura, escrita e cálculos (transtorno de aprendizagem), 6,7\% sintomas de irritabilidade e comportamentos desafiadores, 4,2\% depressão, 5,9\% têm ansiedade com a separação da figura de apego e 4,2\% encontram-se em exposição social (CUNHA, 2017; NASCIMENTO, 2014). O primeiro acolhimento do paciente no CAPSi é o momento do diagnóstico o qual é realizado pela análise das características psicológicas e dos aspectos ambientais ou biológicos do paciente, sendo difícil e duvidoso, visto que a detecção é prejudicada pela forma como o indivíduo é abordado e julgado, pela variabilidade na manifestação clínica dos transtornos e pela alta prevalência de sobreposição de respostas comportamentais e emocionais adaptativas (LEITE, 2016; DSM, 2014).

A avaliação psíquica inicia no consultório e o diagnóstico é definido com base na classificação que utiliza o Manual Diagnóstico e Estatístico de Transtornos Mentais V (DSM V) e a Classificação de Transtornos Mentais e de Comportamento da Classificação Internacional de Doenças e de Problemas Relacionados à Saúde (CID-10) (CARVALHO, 2012). Assim, é possível classificar três grupos diagnósticos: desordens emocionais (internalizantes: depressão, ansiedade, desordens obsessivo-compulsivas); de comportamento disruptivo (externalizantes: conduta desafiadora excessiva, agressividade e comportamento transgressor) e; transtornos do desenvolvimento (problemas de aprendizagem, autismo, entre outros) (VINOCUR, 2011). Essa forma de diagnóstico apresenta limitações quanto a perspectivas culturais e outros aspectos do desenvolvimento negligenciados. $\mathrm{O}$ acompanhamento do paciente deverá ser realizado pela equipe de saúde e família, conforme o 


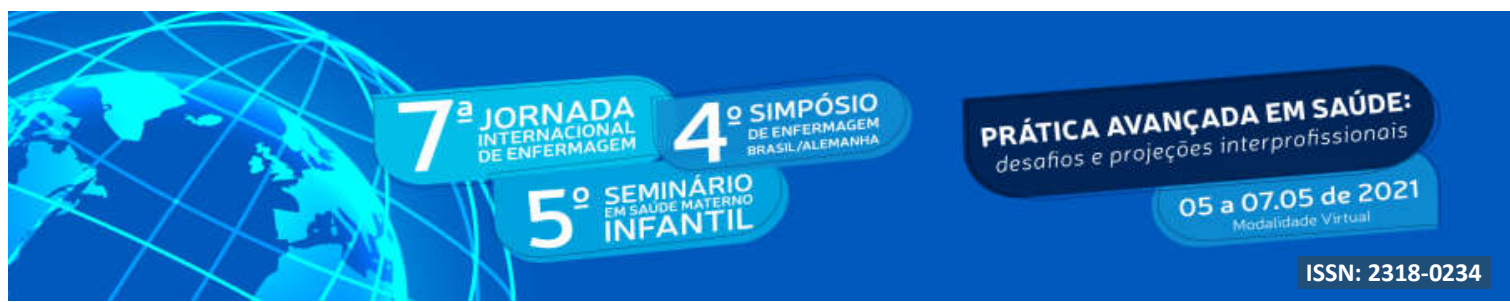

fluxograma baseado no Protocolo de Saúde Mental do município de Florianópolis, Santa Catarina (BRASIL, 2010).

Segundo a OMS, o tratamento implica na adoção de três componentes fundamentais: psicoterapia, farmacoterapia e reabilitação psicossocial integradas (CARVALHO, 2012). Considerando a farmacoterapia, utiliza-se fármacos psicotrópicos, divididos em quatro categorias: ansiolíticos/hipnóticos (2,8\%); antidepressivos $(28,5 \%)$; antiepilépticos $(22,2 \%)$; antipsicóticos/neurolépticos (37,5\%) (LEITE, 2016; CARVALHO, 2014). Outras classes de fármacos disponíveis no CAPSi são os anti-histamínicos $\mathrm{H}_{1}$ (exemplo prometazina associada ao haloperidol, é usada para reduzir agitação e gerar sedação) e estimulantes do Sistema Nervoso Central (SNC, exemplo metilfenidato, que é utilizado no tratamento do Transtorno de Déficit de Atenção com Hiperatividade (TDAH)). Tanto a prometazina quanto o metilfenidato, embora utilizados com frequência não são referenciados quanto a incidência/recorrência de uso (LEITE, 2016; CARVALHO, 2012; CLARK, 2016; MATOS, 2017). Segundo o levantamento bibliográfico realizado, os principais medicamentos prescritos nos CAPSi, baseados no CID-10 são descritos no Quadro 1.

Quadro 1. Principais medicamentos prescritos no CAPSi e dose usual.

\begin{tabular}{|c|c|c|c|}
\hline CID-10 & $\begin{array}{c}\text { CLASSE } \\
\text { FARMACOLÓGICA }\end{array}$ & MEDICAMENTO & DOSE USUAL \\
\hline \multirow{3}{*}{$\begin{array}{l}\text { F20-29 Esquizofrenia, } \\
\text { transtornos } \\
\text { esquizotípicosetranstornos } \\
\text { delirantes }\end{array}$} & $\begin{array}{l}\text { Antipsicóticos e/ou } \\
\text { Neurolépticos }\end{array}$ & Haloperidol & $0,25 \mathrm{mg}$ a $6 \mathrm{mg}$ \\
\hline & & Clorpromazina & $10 \mathrm{mg}$ a $20 \mathrm{mg}$ \\
\hline & & Risperidona & $2 \mathrm{mg}$ a $6 \mathrm{mg} / \mathrm{dia}$ \\
\hline \multirow[t]{6}{*}{$\begin{array}{c}\text { F31 Transtorno afetivo } \\
\text { Bipolar }\end{array}$} & $\begin{array}{c}\text { Estabilizadores de } \\
\text { Humor }\end{array}$ & Lítio & Até $25 \mathrm{Kg}: 600 \mathrm{mg} / \mathrm{dia}$ \\
\hline & & & 25-40 Kg: 900 mg/dia \\
\hline & & & $40-50 \mathrm{Kg}: 1,200 \mathrm{mg} / \mathrm{dia}$ \\
\hline & & & $50-60 \mathrm{~kg}: 1,500 \mathrm{mg} / \mathrm{dia}$ \\
\hline & Antiepilépticos & Carbamazepina & $200 \mathrm{mg}$ a $600 \mathrm{mg}$ \\
\hline & & Ácido Valpróico & $15 \mathrm{mg}$ a $60 \mathrm{mg} / \mathrm{Kg} / \mathrm{dia}$ \\
\hline \multirow[t]{3}{*}{ F32 Episódios depressivos } & ISRS & Fluoxetina & $10 \mathrm{mg}$ a $20 \mathrm{mg}$ \\
\hline & & Sertralina & $25 \mathrm{mg}$ a $200 \mathrm{mg}$ \\
\hline & & Paroxetina & $20 \mathrm{mg}$ a $40 \mathrm{mg}$ \\
\hline F32 Episódios depressivos & Tricíclicos & Imipramina & Dose máxima 5 mg/Kg/dia \\
\hline
\end{tabular}




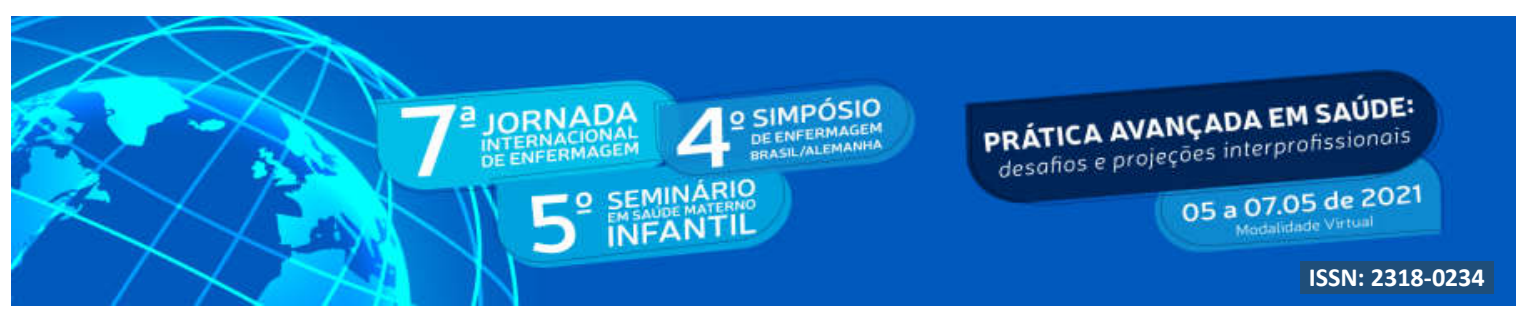

\begin{tabular}{|c|c|c|c|}
\hline & & Clomipramina & Dose máxima 3 mg/dia \\
\hline \multirow[t]{7}{*}{$\begin{array}{c}\text { F41-43 Transtornos de } \\
\text { Ansiedade }\end{array}$} & Benzodiazepínicos & Clonazepam & $\begin{array}{c}\text { Até } 30 \mathrm{Kg} \\
\text { Dose máxima de 0,05 } \\
\mathrm{mg} / \mathrm{Kg} / \mathrm{dia}\end{array}$ \\
\hline & & & $\begin{array}{c}\text { Entre } 10 \text { e } 16 \text { anos } \\
\text { Dose máxima de } 3 \mathrm{mg} \text { a } 6 \\
\mathrm{mg} / \mathrm{dia}\end{array}$ \\
\hline & ISRS & Fluoxetina & $10 \mathrm{mg}$ a $20 \mathrm{mg}$ \\
\hline & & Sertralina & $25 \mathrm{mg}$ a $200 \mathrm{mg}$ \\
\hline & & Paroxetina & $20 \mathrm{mg}$ a $40 \mathrm{mg}$ \\
\hline & Tricíclicos & Imipramina & Dose máxima $5 \mathrm{mg} / \mathrm{Kg} / \mathrm{dia}$ \\
\hline & & Clomipramina & Dose máxima 3 mg/dia \\
\hline \multirow{5}{*}{$\begin{array}{l}\text { F84 Transtornos globais } \\
\text { do desenvolvimento }\end{array}$} & ISRS & Fluoxetina & $10 \mathrm{mg}$ a $20 \mathrm{mg}$ \\
\hline & & Sertralina & $25 \mathrm{mg}$ a $200 \mathrm{mg}$ \\
\hline & & Paroxetina & $20 \mathrm{mg}$ a $40 \mathrm{mg}$ \\
\hline & $\begin{array}{l}\text { Antipsicóticos e/ou } \\
\text { Neurolépticos }\end{array}$ & Haloperidol & $0,25 \mathrm{mg}$ a $6 \mathrm{mg}$ \\
\hline & & Risperidona & $2 \mathrm{mg}$ a $6 \mathrm{mg} / \mathrm{dia}$ \\
\hline
\end{tabular}

ISRS: Inibidor seletivo da recaptação de serotonina. Fonte: Do autor.

Uma dificuldade existente na prática psiquiátrica é a escolha das medicações para os diversos quadros clínicos, sendo comum a associação de psicofármacos de diferentes classes, gerando a polifarmácia e interações medicamentosas (Tabela 2) (CARVALHO, 2015; GOMES, 2013).

Tabela 2. Possíveis interações medicamentosas e os efeitos provocados.

\begin{tabular}{|c|c|c|c|c|c|c|c|c|c|c|c|c|c|c|c|}
\hline & MEDICAMENTO & $\begin{array}{c}1 \\
\mathrm{AV} \\
\end{array}$ & $\begin{array}{c}2 \\
\text { Carb } \\
\end{array}$ & $\begin{array}{c}3 \\
\mathrm{CL} \\
\end{array}$ & $\begin{array}{c}4 \\
\text { Clo } \\
\end{array}$ & \begin{tabular}{|c|}
5 \\
Clon \\
\end{tabular} & $\begin{array}{c}6 \\
\text { Clor } \\
\end{array}$ & $\begin{array}{c}7 \\
\text { Fluo }\end{array}$ & \begin{tabular}{|c|}
8 \\
Halo \\
\end{tabular} & $\begin{array}{c}9 \\
\text { Imip }\end{array}$ & \begin{tabular}{|c|}
10 \\
Met
\end{tabular} & $\begin{array}{c}11 \\
\text { Paro } \\
\end{array}$ & $\begin{array}{c}12 \\
\text { Pro }\end{array}$ & $\begin{array}{l}13 \\
\text { Ser }\end{array}$ & $\begin{array}{c}14 \\
\text { Risp }\end{array}$ \\
\hline 1 & $\begin{array}{l}\text { Ácido Valpróico } \\
\text { (AV) }\end{array}$ & & $\uparrow$ & & & & * & & & & & & $\varnothing$ & & $\uparrow$ \\
\hline 2 & $\begin{array}{l}\text { Carbamazepina } \\
\text { (Carb) }\end{array}$ & $\downarrow$ & & N & $\downarrow$ & $\downarrow$ & $\leftrightarrow$ & & $\downarrow$ & & & & & & $\downarrow$ \\
\hline 3 & $\begin{array}{l}\text { Carbonato de } \\
\text { Lítio (CL) }\end{array}$ & & $\mathrm{A}$ & & & & $\begin{array}{l}\S ; \downarrow ; \\
\diamond ;: \propto\end{array}$ & $\begin{array}{l}\uparrow ; \\
*\end{array}$ & $\begin{array}{l}\diamond ; \\
\alpha\end{array}$ & & & $* ;$ & $\begin{array}{l}\nabla ; \\
\infty\end{array}$ & $\begin{array}{l}* ; \\
\square\end{array}$ & $\begin{array}{l}\diamond ; \\
a\end{array}$ \\
\hline 4 & $\begin{array}{l}\text { Clomipramina } \\
\text { (Clo) }\end{array}$ & $\uparrow$ & $\downarrow$ & & & & & $\begin{array}{l}\mathrm{x} \\
\leftrightarrow \\
*\end{array}$ & $\leftrightarrow$ & & & & $\leftrightarrow$ & & \\
\hline 5 & $\begin{array}{c}\text { Clonazepam } \\
\text { (Clon) }\end{array}$ & & $\downarrow$ & & & & & & & & & & & & \\
\hline 6 & $\begin{array}{l}\text { Clorpromazina } \\
\text { (Clor) }\end{array}$ & & & $\begin{array}{l}\diamond \\
\infty\end{array}$ & & & & $\leftrightarrow$ & $\begin{array}{l}; \\
\Delta ; \\
\leftrightarrow\end{array}$ & & & & $\stackrel{\leftrightarrow}{\Delta}$ & & $\bullet$ \\
\hline 7 & Fluoxetina (Fluo) & * & $\uparrow$ & * & $\begin{array}{l}\mathrm{x} \\
\stackrel{\leftrightarrow}{\leftrightarrow} \\
*\end{array}$ & & $\stackrel{\uparrow ;}{\leftrightarrow}$ & & $\begin{array}{c}\uparrow ; \mathbf{\varphi} \\
\Delta\end{array}$ & *; & $\square$ & & $\begin{array}{l}\mathbf{Y} \\
\mathrm{x}\end{array}$ & & $\leftrightarrow$ \\
\hline
\end{tabular}




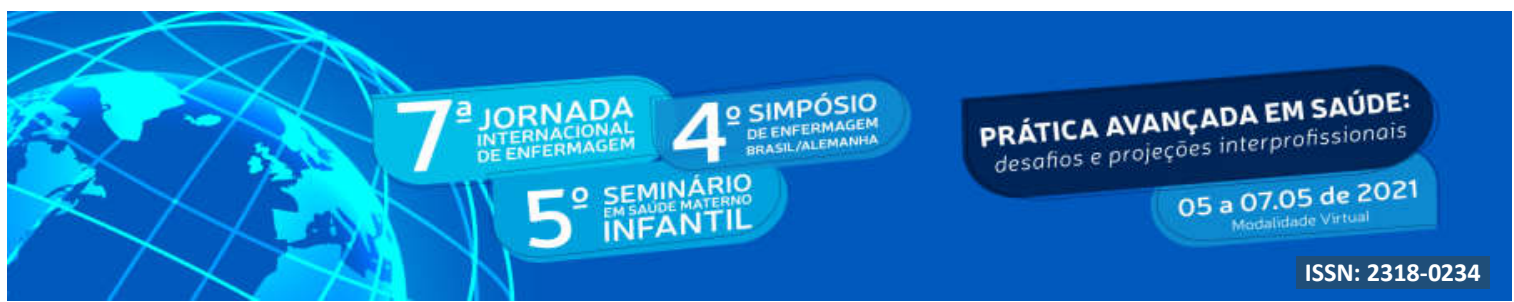

\begin{tabular}{|c|c|c|c|c|c|c|c|c|c|c|c|c|c|c|}
\hline 8 & $\begin{array}{l}\text { Haloperidol } \\
\text { (Halo) }\end{array}$ & & $\downarrow$ & $\begin{array}{c}\uparrow ; \\
\diamond ; a\end{array}$ & $\leftrightarrow$ & $\uparrow ; \Delta ;$ & $\uparrow$ & & $\leftrightarrow$ & & & $\stackrel{\leftrightarrow}{\Delta}$ & $\leftrightarrow$ & $\leftrightarrow$ \\
\hline 9 & Imipramina (Imip) & & & & & & & & & & $* ; \overline{\mathrm{T}}$ & & & \\
\hline 10 & $\begin{array}{l}\text { Metilfenidato } \\
\text { (Met) }\end{array}$ & & & & & & * & & & & * & & $*$ & \\
\hline 11 & Paroxetina (Paro) & & & & $\overline{\mathbf{T}}$ & & & & & & & & & \\
\hline 12 & Prometazina (Pro) & $\varnothing$ & & $\begin{array}{l}\diamond ; \\
\infty\end{array}$ & $\leftrightarrow$ & $\begin{array}{l}\Delta ; \\
\leftrightarrow\end{array}$ & & $\begin{array}{l}\Delta \\
\leftrightarrow \\
\leftrightarrow\end{array}$ & & & & & & \\
\hline 13 & Sertralina (Ser) & & & & $\begin{array}{l}* ; \\
\overline{\mathrm{T}}\end{array}$ & & & & & $\square$ & & & & \\
\hline 14 & $\begin{array}{l}\text { Risperidona } \\
\text { (Risp) }\end{array}$ & & $\downarrow$ & $\diamond ; a$ & & $\vee$ & $\uparrow$ & $\leftrightarrow$ & & & & & & \\
\hline
\end{tabular}

Fonte: Adaptado de Fernandes et al (2012); Carvalho et al (2015).

Legenda:

$\uparrow:$ Pode resultar no aumento da concentração plasmática e/ou da eficácia

$\downarrow$ : Pode resultar na diminuição da concentração plasmática e/ou da eficácia

*: Pode resultar em aumento do risco de síndrome serotoninérgica

Ø: Pode aumentar os riscos de toxicidade no fígado

N: Pode resultar em aditivo de neurotoxicidade

$\diamond$ : Pode resultar em danos cerebrais

$\mathrm{a}:$ Pode resultar em discinesias e/ou fraqueza muscular

$\Delta$ : Pode aumentar os riscos de reações extrapiramidais

$\leftrightarrow$ : Pode resultar em aumento do risco de prolongamento do intervalo QT

४: Pode resultar em um aumento do risco de cardiotoxicidade

$\mathrm{x}$ : Pode resultar em toxicidade

$\S$ : Pode ter seus efeitos tóxicos mascarados

$\overline{\mathrm{T}}$ : Pode aumentar as concentrações plasmáticas de alguns antidepressivos tricíclicos

$\square$ : Pode aumentar os efeitos farmacológicos ISRS

No estudo de Balen et al. (2017) as interações mais recorrentes estão associação entre antidepressivos, confirmando os dados pesquisados. A fluoxetina com tricíclicos, causa risco de cardiotoxicidade, que pode resultar em parada cardíaca, logo, recomenda-se evitar esta associação. No entanto, a combinação é recomendada para o tratamento de depressão persistente, contanto que, monitorado. A fluoxetina com antipsicóticos pode ocasionar risco de prolongamento do intervalo QT (parâmetro eletrocardiográfico que representa a duração da sístole, ou seja, contração cardíaca). Recomenda-se monitorar a arritmia ventricular, descontinuar a fluoxetina e, monitorar a toxicidade da mesma.

Medicamentos que atuam no SNC classificados como de baixo índice terapêutico, 


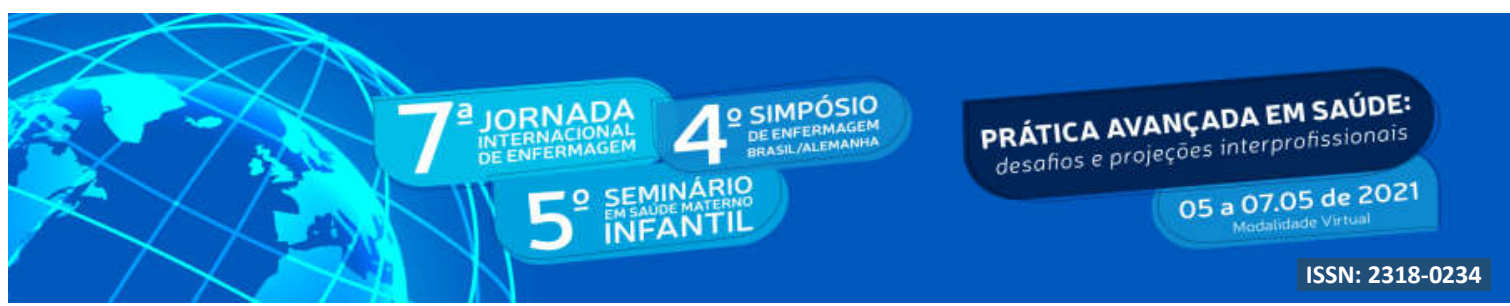

como o carbonato de lítio geram distintas interações quando associado, podendo ser graves (com haloperidol, clorpromazina e risperidona) a moderadas (com fluoxetina e carbamazepina). No uso concomitante com antipsicóticos recomenda-se a redução de dose do antipsicótico ou substituição por outra classe a fim de evitar efeitos adversos. Junto com a fluoxetina aumenta o risco de desenvolvimento de síndrome serotoninérgica, que pode ser fatal (FERNANDES, 2012; BALEN, 2017). O haloperidol pode ter sua ação diminuída pelo fenobarbital, carbamazepina e fenitoína, aumentando as reações adversas quando associado à fluoxetina, lítio, tricíclicos ou medicamentos que causam reações extrapiramidais. Com clorpromazina pode desencadear um prolongamento do intervalo QT e resultar em maior risco de arritmias ventriculares, incluindo torsades de pointes (forma de taquicardia ventricular polimórfica em pacientes com um intervalo QT longo) e morte súbita. Logo, a associação não é recomendada (FERNANDES, 2012; BALEN, 2017).

As interações da clorpromazina compreendem maior ação de antidepressivos tricíclicos, a diminuição de sua ação pelo lítio, riscos de queda da pressão sanguínea e aumento de reações extrapiramidais. Aos associa-la à risperidona, há risco de cardiotoxicidade, sendo o uso concomitante não recomendado. Já a clorpromazina com prometazina, aumenta o risco de prolongamento do intervalo QT, necessitando a monitorização (FERNANDES, 2012; BALEN, 2017). A risperidona aumenta a ação da fenitoína provocando aumento das reações com lítio e a queda na pressão arterial. Nesse caso, recomenda-se alteração de dosagem e, até mesmo, substituição de um dos medicamentos, conforme o estado do paciente (FERNANDES, 2012; BALEN, 2017).

Anticonvulsivantes, como carbamazepina associada a imipramina ou clomipramina, pode ter sua efetividade diminuída, sendo necessário, o ajuste da dose. Junto à risperidona gera diminuição nos níveis plasmáticos referentes à risperidona. Deve-se descontinuar a carbamazepina ou outros indutores de enzimas hepáticas, ou reduzir a dose de risperidona. A associação entre carbamazepina e haloperidol leva a uma diminuição dos níveis plasmáticos do antipsicótico, com perda de eficácia terapêutica, devendo-se monitora-la (BALEN, 2017).

Ácido valpróico e antipsicóticos podem promover o aumento da sedação e delírios. Já com a risperidona, a associação pode resultar em concentrações aumentadas do ácido valpróico, sendo necessário monitorar os seus níveis e os de amônia (FERNANDES, 2012; 


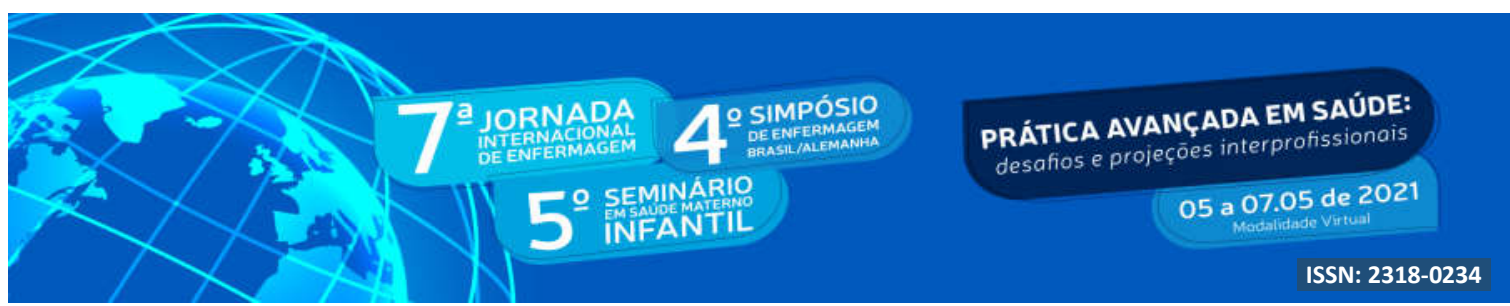

BALEN, 2017). E, a prometazina ao ser associada com o haloperidol pode aumentar os riscos de reações extrapiramidais e toxicidade hepática (FERNANDES, 2012; BALEN, 2017).

No panorama brasileiro existem poucos estudos sobre interações medicamentosas, sendo restritos a quantificar frequência, sem informações dos cuidados e, também não há uma base de dados computadorizada integrando interações entre os psicofármacos (GOMES, 2013). Se faz necessário propor um acompanhamento farmacoterapêutico, o qual se baseará em adaptações do Método Dáder, o qual envolve uma entrevista com paciente e/ou familiar onde avaliam-se todos os medicamentos que utiliza e já utilizou e exames laboratoriais, considerando as dúvidas em relação a saúde, medicamento e PRM (GOMES, 2013; HERNÁNDEZ, 2010). Elabora-se um documento com a história farmacoterapêutica do paciente, comorbidades e orienta-se quanto as interações medicamentosas. A seguir, é analisado se o medicamento é necessário, eficaz, seguro e os possíveis PRM, o que permite a intervenção farmacêutica, com ajuste de dosagens, alteração de horários e indicação de substituição de medicamentos de mesma classe terapêutica, sendo opcional a adesão do prescritor (GOMES, 2013; HERNÁNDEZ, 2010). Após entrevistas farmacêuticas sucessivas, o acompanhamento farmacoterapêutico é finalizado, analisando se as intervenções surtiram resultados (GOMES, 2013; HERNÁNDEZ, 2010).

Logo, sugere-se a alteração do método Dáder com a inclusão, na primeira etapa de anamnese, de aspectos sociodemográficos, práticas disciplinares adotadas pelos pais, discórdia conjugal, história de transtornos psiquiátricos dos pais, presença de doença física capaz de trazer limitações às crianças, problemas hereditários, desordens cerebrais, eventos estressantes agudos, questões religiosas e laborais, informações sobre o parto, adoção e fatores ambientais que podem ser desencadeadores dos transtornos mentais, de forma a contribuir para o diagnóstico e acompanhamento do paciente (THIENGO, 2014).

\section{CONCLUSÃO}

$\mathrm{O}$ atendimento dos CAPS exige a intervenção farmacêutica como um serviço conciliador da terapêtica para identificar e prevenir os potenciais PRMs em poli terapias infantojuvenis, principalmente por interferir no desenvolvimento da criança e adolescente. 


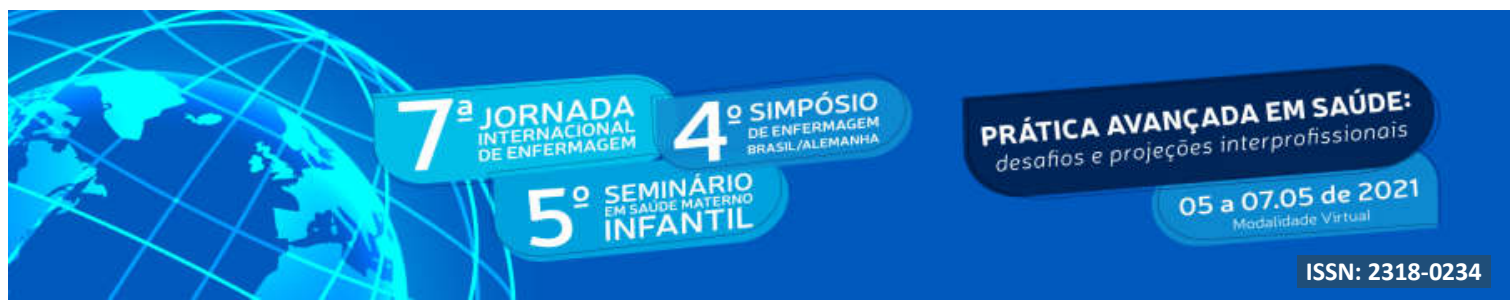

\section{REFERÊNCIAS}

BALEN, E. et al. Interações medicamentosas potenciais entre medicamentos psicotrópicos dispensados. Jornal Brasileiro de Psiquiatria, v. 66, n. 3, p. 172-7, Jul-set. 2017.

BRASIL. Ministério da Saúde. Secretaria de Atenção à Saúde. Atenção psicossocial a crianças e adolescentes no SUS: tecendo redes para garantir direitos. Brasília, 2014. Disponível

em: http://bvsms.saude.gov.br/bvs/publicacoes/atencao_psicossocial_criancas_adolescentes_sus.p df. Acesso em: 15/03/2021. 59p.

BRASIL. Ministério da Saúde. Secretaria de Atenção à Saúde. Centros de Atenção Psicossocial e Unidades de Acolhimento como lugares da atenção psicossocial nos territórios: orientações para elaboração de projetos de construção, reforma e ampliação de CAPS e de UA/Ministério da Saúde. Brasília: 2015. Disponível em: http://bvsms.saude.gov.br/bvs/publicacoes/centros_atencao_psicossocial_unidades_acolhimen to.pdf. Acesso em: 1506/2021. 46p.

CARVALHO, I. L. N. Uso de psicofármacos em adolescentes atendidos pelos centros de atenção psicossocial infantojuvenil de Fortaleza-CE. 2012. 106 p. Dissertação (Mestrado em Saúde Coletiva). Universidade de Fortaleza, Ceará, 2012.

CARVALHO, I. L. N. et al. CAPSi: avanços e desafios após uma década de funcionamento. Cadernos Brasileiros de Saúde Mental, v. 6, n.14, p.42-60, Jul-fev, 2014.

CARVALHO, I. B. et al. Estudo das potenciais interações de medicamentos sujeitos a controle especial em um Centro de Atenção Psicossocial (CAPS) no município de Teresina PI. Boletim Informativo Geum, v.6, n.1, p.7-15, Jan-mar. 2015.

CLARK, M. A. et al. Farmacologia ilustrada. 6. ed. Porto Alegre: Artmed, 2016. 680p.

CUNHA, M. P.; BORGES, L. M.; BEZERRA, C. B. Infância e Saúde mental: perfil das crianças usuárias de um Centro de Atenção Psicossocial Infantil. Mudanças - Psicologia da Saúde, v. 25, n. 1, p.27-35, Jan-jun. 2017.

DSM - V. Manual Diagnóstico e Estatístico de Transtornos Mentais. 5 ed. Porto Alegre: Artmed, 2014. 992 p.

FERNANDES, M. A. et al. Interações medicamentosas entre psicofármacos em um serviço especializado de saúde mental. Revista Interdisciplinar NOVAFAPI, v. 5, n. 1, p. 9-15, Janmar. 2012.

GOMES, E. F. Importância da assistência e da atenção farmacêutica aplicada a pacientes com transtornos mentais. 2013. 86 p. Trabalho de Conclusão de Curso, Curso de Farmácia, Faculdade Católica Salesiana do Espírito Santo, Vitória (ES), 2013. 


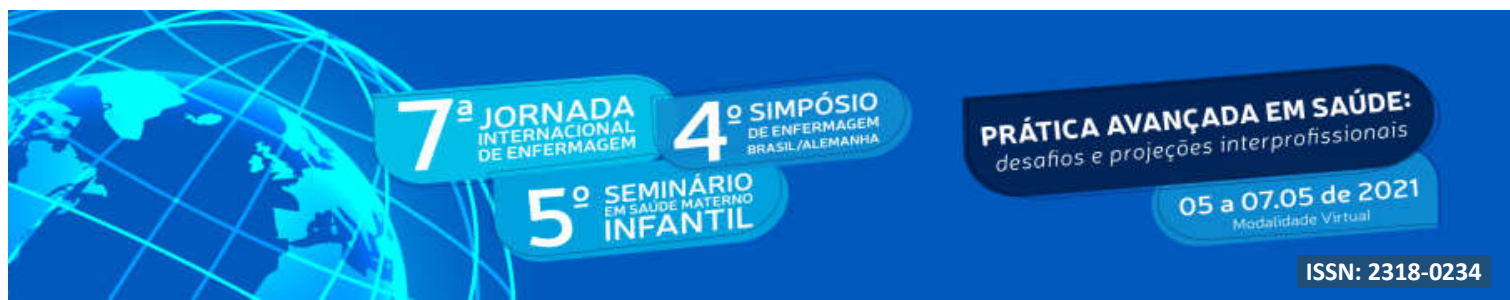

HERnÁndeZ, D. S.; CASTRO, M. M. S.; DÁDER, M. J. F. Método Dáder: Manual de Seguimento Farmacoterapêutico. Lisboa: Grupo de Investigación em Atención Farmacéutica (CTS-131), 2010. 128p.

HIRDES, A. A reforma psiquiátrica no Brasil: uma (re)visão. Ciência \& Saúde Coletiva, v.14, n. 1, p.297-305, out, 2009.

LEITE, L. O. B. et al. Os principais medicamentos prescritos em centros de apoio psicossocial: CAPs. INTESA - Informativo Técnico do Semiárido, Pombal-PB, v.10, n. 2, p.76-91, Jul-dez, 2016.

LOURENÇO, L. Transtornos mentais atingem 23 milhões de pessoas no Brasil. Brasília: jun. 2010. Disponível em: http://memoria.ebc.com.br/agenciabrasil/noticia/2010-0628/transtornos-mentais-atingem-23-milhoes-de-pessoas-no-brasil. Acesso em: 30/10/2018.

MATOS, R. K. S. et al. Projeto terapêutico singular em um centro de atenção psicossocial (Caps II). Revista Intercâmbio Unimontes, v.9, p.111-30, dez. 2017.

NASCIMENTO, Y. C. M. L. et al. Perfil de crianças e adolescentes acompanhados por um centro de atenção psicossocial infanto-juvenil. Revista de Enfermagem UFPE online, v. 8, n.5, p.1261-72, maio. 2014.

SANTA CATARINA (Estado). Secretaria Municipal de Saúde. Protocolo de atenção em saúde mental. Tubarão: Copiart, 2010.

TAVARES, S. F. V. Vivência do atendimento no centro de atenção psicossocial infantojuvenil: motivações da família e profissionais. 2012. p.3467-72. Dissertação (Mestrado em Cuidados Clínicos em Enfermagem e Saúde). Universidade Estadual do Ceará, Fortaleza, 2018.

ThIENGO, D. L.; CAVAlCANTE, M. T.; LOVISI, G. M. Prevalência de transtornos mentais entre crianças e adolescentes e fatores associados: uma revisão sistemática. Jornal Brasileiro de Psiquiatria, v. 63, n.4, p.360-72, Out-dez. 2014.

VIEIRA, C. M. A. Perfil dos usuários do Centro de Atenção Psicossocial: Taguatinga - DF. 2013. p.161-75. Trabalho de Conclusão de Curso, Curso de Terapia Ocupacional, Faculdade de Ceilândia, Universidade de Brasília, Brasília (DF). 2013.

VINOCUR, E.; PEREIRA, H. V. F. S. Avaliação dos transtornos de comportamento na infância. Revista Hospital Universitário Pedro Ernesto, UERJ, ano 10, p. 26-34, ago. 2011. 